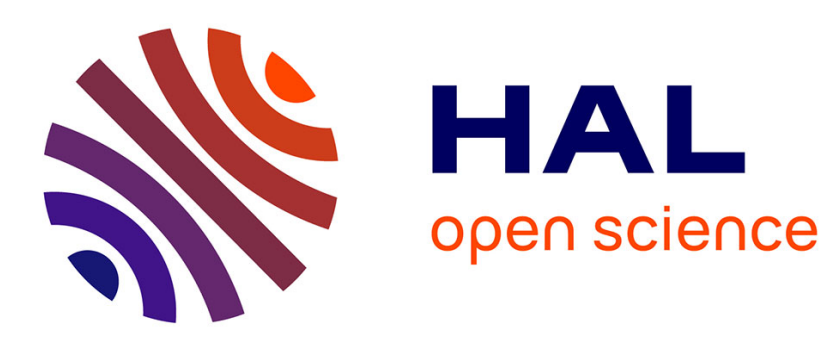

\title{
On the stability analysis of multiple model systems
}

\author{
Mohammed Chadli, Didier Maquin, José Ragot
}

\section{To cite this version:}

Mohammed Chadli, Didier Maquin, José Ragot. On the stability analysis of multiple model systems.

European Control Conference, ECC'2001, Sep 2001, Porto, Portugal. pp.CDROM. hal-00278221

\section{HAL Id: hal-00278221 \\ https://hal.science/hal-00278221}

Submitted on 9 May 2008

HAL is a multi-disciplinary open access archive for the deposit and dissemination of scientific research documents, whether they are published or not. The documents may come from teaching and research institutions in France or abroad, or from public or private research centers.
L'archive ouverte pluridisciplinaire HAL, est destinée au dépôt et à la diffusion de documents scientifiques de niveau recherche, publiés ou non, émanant des établissements d'enseignement et de recherche français ou étrangers, des laboratoires publics ou privés. 


\title{
ON THE STABILITY ANALYSIS OF MULTIPLE MODEL SYSTEMS
}

\author{
Mohammed CHADLI, Didier MAQUIN, José RAGOT
}

\author{
Centre de Recherche en Automatique de Nancy, CNRS, UPRESA 7039 \\ 2, Avenue de la forêt de Haye, 54516 Vandoeuvre les Nancy - France. \\ Phone: (33) 3835956 84, Fax: (33) 383595644 \\ Email: \{Mohamed.Chadli, Didier.Maquin, José.Ragot\}@ensem.inpl-nancy.fr
} Keywords: Multiple model approach, nonlinear systems,
controllers, observers, stability.

\begin{abstract}
This paper studies the global asymptotic stability of the nonlinear systems described by the multiple model approach using the Takagi-Sugeno (T-S) fuzzy modelling. New sufficient conditions for the stability of such systems are given. Stability analysis is derived via Lyapunov technique and LMIs (Linear Matrix Inequalities) formulation obtained from BMIs (Bilinear Matrix Inequalities) linearisation. Following a similar approach, sufficient conditions to guarantee the stability of T-S fuzzy systems for both T-S fuzzy controllers and T-S fuzzy observers are derived. The stabilisation of the closed loop continuous T-S fuzzy models is discussed using the well-known PDC (Parallel Distributed Compensation) technique.
\end{abstract}

\section{Introduction}

The issue of stability and stabilisation of Takagi-Sugeno (TS) fuzzy control systems has been considered extensively in nonlinear frameworks. The major advantage of the modelling approach proposed by T-S (or Takagi-Sugeno-Kang) [5][6] is the fact that this one has been shown to be a universal approximator [9][14][17]. Universal approximator means that given any real-valued continuous function on a compact subset of $\mathbb{R}^{n}$, there is a T-S fuzzy model that will approximate this function to any accuracy [14]. Universal controllers mean that given any process that can be controlled by a continuous-time controller, it can also be controlled by a T-S fuzzy controller [14]. The T-S fuzzy models are described by a set of fuzzy "If ... then" rules with fuzzy sets in the antecedents and dynamic LTI systems in the consequent. These sub-models are considered as local linear models, the aggregation of which represent the nonlinear system behaviour.

For example, Tanaka and Sugeno presented sufficient conditions for the stability of T-S models [19] using a quadratic Lyapunov approach. The stability depends on the existence of a common positive definite matrix guarantying the stability of all local subsystems. These stability conditions may be expressed in linear matrix inequalities (LMIs) form [20]. The obtaining of a solution is then facilitated by using numerical toolboxes for solving such problems. Recently LMI constraint has been added to compute a decay rate and to guarantee that the control law action does not permit an exceeding of a pre-defined norm-bound of input and output signals [2][21]. LMIs constraints have also been used for pole assignment in LMI regions to achieve the desired fuzzy controllers and fuzzy observer's performances [16][18]. However, if the number of sub-models is large, it might be difficult to find a common matrix that simultaneously stabilises all the local models. Moreover, these constraints are often very conservative and it is well known that, in a lot of cases, such a common positive definite matrix does not exist, whereas the system is stable.

To overcome this limitation, some works have been developed in order to establish new stability conditions by relaxing some of the previous constraints. So one way for obtaining relaxed stability conditions consists to use a piecewise quadratic Lyapunov function formulated as a set of LMIs [22]. Using the PI fuzzy controller and the Lyapunov technique, the authors in [23] show that asymptotic stability of the Takagi-Sugeno fuzzy systems can be ensured under certain restrictions on the control signal and the rate of change of the output. Jadbabaie in [24] uses a non-quadratic Lyapunov function to prove the stability of the T-S fuzzy systems by fixing an a priori bound on the variation of the state. In [15] using LMIs technique and non-quadratic Lyapunov function, a systematic way to pick the bound of state variable vector is derived to prove the stability and the stabilisability of the T-S fuzzy systems.

Using the Lyapunov technique and LMI formulation, the aim of this paper is to derive new sufficient conditions for stability of the T-S fuzzy models and also for both T-S fuzzy controllers and T-S fuzzy observers. The stabilization is discussed using the PDC technique [1][2][13].

This paper is organised as follows: section 2 presents an overview of T-S fuzzy systems. In section 3 we recall the LMI formulation of basic quadratic stability conditions. In section 4, we develop new sufficient conditions to prove the stability of T-S continuous fuzzy systems. In sections 5 and 6 , using the concept of PDC to perform control laws, a similar approach is used for the design of fuzzy controllers and fuzzy 
observers. For solving the obtained BMIs problem, a linearisation method is used.

\section{Notation}

In this paper, we denote the minimum and maximum eigenvalues of the matrix $X$ by $\lambda_{\text {min }}(X)$ and $\lambda_{\text {Max }}(X)$, the conjugate transpose of $X$ by $X^{*}$, the transpose of $X$ by $X^{T}$. The Hermitian and non Hermitian parts of a matrix $X$, respectively denoted by $X_{h}$ and $X_{\hbar}$, are defined as follows, $X_{h}=\frac{1}{2}\left(X+X^{*}\right), X_{\hbar}=\frac{1}{2}\left(X-X^{*}\right)$.

$\lambda_{k}(X)$ is the $k^{\text {th }}$ eigenvalue of the matrix $X$.

\section{Continuous Takagi-Sugeno systems}

The T-S fuzzy models are described by a set of fuzzy "If...then" rules with fuzzy sets in the antecedents and dynamic LTI systems in the consequent. These sub-systems represent local linear input-output relations of a non-linear system. A general T-S plant rule can be written as follows:

$i^{\text {th }}$ rule:

$$
\begin{gathered}
I F z_{1}(t) \text { is } F_{1}^{i} \text { and... and } z_{q}(t) \text { is } F_{q}^{i} \\
\text { THEN } \dot{x}(t)=A_{i} x(t)+B_{i} u(t)
\end{gathered}
$$$$
\forall i \in\{1, \ldots, n\}
$$

where $n$ is the number of rules (sub-models), $x(t) \in \mathbb{R}^{p}$ is the state vector, $y(t) \in \mathbb{R}^{l}$ is the output vector, $u(t) \in \mathbb{R}^{m}$ is the input vector, $A_{i} \in \mathbb{R}^{p x p}, B_{i} \in \mathbb{R}^{p x m}, z(t) \in \mathbb{R}^{q}$ is the premise variable, depending on the measurable state variables and, possibly, on the input.

The global T-S fuzzy system is inferred as follows:

$\dot{x}(t)=\frac{\sum_{i=1}^{n} \omega_{i}(z(t))\left(A_{i} x(t)+B_{i} u(t)\right)}{\sum_{i=1}^{n} \omega_{i}(z(t))}$

where $\omega_{i}(z(t))=\prod_{j=1}^{q} f_{j}^{i}\left(z_{j}(t)\right)$

and $f_{j}^{i}\left(z_{j}(t)\right)$ is the membership grade of $z_{j}(t)$ to the fuzzy set $F_{j}^{i}$.

Finally we define:

$$
\mu_{i}(z(t))=\frac{\omega_{i}(z(t))}{\sum_{i=1}^{n} \omega_{i}(z(t))}
$$

$\mu_{i}(z(t))$ is the normalised membership function in relation with the $\mathrm{i}^{\text {th }}$ sub-system such that:

$$
\left\{\begin{array}{l}
\sum_{i=1}^{n} \mu_{i}(z(t))=1 \\
0 \leq \mu_{i}(z(t)) \leq 1 \quad \forall i \in\{1, \ldots, n\}
\end{array}\right.
$$

Equation (1) can be rewritten as:

$\dot{x}(t)=\sum_{i=1}^{n} \mu_{i}(z(t))\left(A_{i} x(t)+B_{i} u(t)\right)$

The final output of T-S fuzzy model is also inferred as follows:

$$
\begin{gathered}
y(t)=\frac{\sum_{i=1}^{n} \omega_{i}(z(t)) C_{i} x(t)}{\sum_{i=1}^{n} \omega_{i}(z(t))}, \text { that is } \\
y(t)=\sum_{i=1}^{n} \mu_{i}(z(t)) C_{i} x(t)
\end{gathered}
$$

\section{Basic stability conditions}

The open-loop T-S fuzzy system of (4) is defined as:

$\dot{x}(t)=\sum_{i=1}^{n} \mu_{i}(z(t)) A_{i} x(t)$

Let us recall first the basic stability conditions of the openloop system (6) derived using quadratic Lyapunov function.

The continuous fuzzy system described by (6) is globally asymptotically stable if there exists a common matrix $P=P^{T}>0$ such that [19]:

$A_{i}^{T} P+P A_{i}<0 \quad \forall i \in\{1, \ldots, n\}$

The existence of such a common positive definite matrix described by LMIs (7) is a key to check the global stability of a $\mathrm{T}-\mathrm{S}$ continuous fuzzy system.

The authors in [10] propose an analytic way to finding a common $P$ in the particular case where the matrices $A_{i}$ are asymptotically stable and commute pairwise.

For a given positive definite matrix $Q$, let $P_{1}, \ldots, P_{n}$ be the unique solution of the following Lyapunov equations:

$A_{1}^{T} P_{1}+P_{1} A_{1}=-Q$
$A_{i}^{T} P_{i}+P_{i} A_{i}=P_{i-1} \quad \forall i \in\{2, \ldots, n\}$

If the following assumption is made:

$A_{i} A_{j}=A_{j} A_{i} \quad \forall i, j \in\{1, \ldots, n\}$ 
i.e. all matrices commute pairwise, then the T-S fuzzy system (6) is globally asymptotically stable and have as a common lyapunov function $V(x)=x^{T} P_{n} x[10]$.

These results mean that a sufficient condition for the existence of a common $P$ is that each $A_{i}$ must be asymptotically stable and commute pairwise.

It is also shown that a common $P$ exists only if the following conditions hold [8]:

$A_{i}+A_{j}<0 \quad \forall i, j \in\{1, \ldots, n\}$

The proof can be easily obtained by summing the LMIs (7). This result means that a necessary condition for the existence of a common $P$ is that each $A_{i}$ and $\left(A_{i}+A_{j}\right)$ must be stable.

These above conditions show clearly the conservativeness of the method, i.e. we can find T-S fuzzy systems that are stable but for which there is no common $P$ to prove the stability.

\section{Stability analysis}

In this section, we present a new sufficient condition for the global asymptotic stability of an unforced continuous T-S fuzzy model (6).

We need the following theorems to prove Theorem 3

Theorem 1 [7]: Weyl inequality

Let $X$ and $Y$ be Hermitian matrices. Then the eigenvalues of $X+Y$ are such that $\forall k \in\{1, \ldots, s\}$

$\lambda_{\text {min }}(Y)+\lambda_{k}(X) \leq \lambda_{k}(X+Y) \leq \lambda_{k}(X)+\lambda_{\max }(Y)$

Where

$\lambda_{\text {min }}(X)=\lambda_{1}(X) \leq . \leq \lambda_{k}(X) \leq . \leq \lambda_{s}(X)=\lambda_{\text {max }}(X)$

Theorem 2 [25]

Let $X$ be square matrix. $X_{h}$ and $X_{\hbar}$, the Hermitian and nonHermitian matrices related to $X$. Then $\forall k \in\{1, \ldots, s\}$

$\left\{\begin{array}{l}\lambda_{\min }\left(X_{h}\right) \leq \mathfrak{R} e\left(\lambda_{k}(X)\right) \leq \lambda_{\max }\left(X_{h}\right) \\ \min \left(\frac{1}{J}\left(\lambda\left(X_{\hbar}\right)\right)\right) \leq \mathfrak{I} m\left(\lambda_{k}(X)\right) \leq \max \left(\frac{1}{J}\left(\lambda\left(X_{\hbar}\right)\right)\right)\end{array}\right.$

Where $J^{2}=-1$.

Considering the unforced system (6) rewritten as $\dot{x}(t)=A(z(t)) x(t)$, where $A(z(t))=\sum_{i=1}^{n} \mu_{i}(z(t)) A_{i}$ the Hermitian and non Hermitian matrices of $A(z), A_{h}(z)$ and $A_{\hbar}(z)$, can be expressed as follows
$A_{h}(z(t))=\sum_{i=1}^{n} \mu_{i}(z(t)) A_{i h}$
$A_{\hbar}(z(t))=\sum_{i=1}^{n} \mu_{i}(z(t)) A_{i \hbar}$

Taking into account the properties of the normalised membership function (3), it directly follows from theorem 1 that

$\left\{\begin{array}{l}\sum_{\substack{i=1 \\ i \neq p}}^{n} \mu_{i}(z(t)) \lambda_{\text {min }}\left(A_{i h}\right)+\mu_{p}(z(t)) \lambda_{k}\left(A_{p h}\right) \leq \lambda_{k}\left(A_{h}(z(t))\right) \\ \lambda_{k}\left(A_{h}(z(t))\right) \leq \mu_{p}(z(t)) \lambda_{k}\left(A_{p h}\right)+\sum_{\substack{i=1 \\ i \neq p}}^{n} \mu_{i}(z(t)) \lambda_{\text {max }}\left(A_{i h}\right)\end{array}\right.$
$\forall i, p \in\{1, \ldots, n\} \quad \forall k \in\{1, \ldots, s\}$

Theorem 2 applied to (10) allows the following inequality to be deduced.

$$
\begin{aligned}
& \left\{\begin{array}{l}
\sum_{i=1}^{n} \mu_{i}(z(t)) \lambda_{\text {min }}\left(A_{i h}\right) \leq \Re e\left(\lambda_{k}(A(z(t)))\right) \\
\Re e\left(\lambda_{k}(A(z(t)))\right) \leq \sum_{i=1}^{n} \mu_{i}(z(t)) \lambda_{\text {max }}\left(A_{i h}\right)
\end{array}\right. \\
& \forall k \in\{1, \ldots, s\}
\end{aligned}
$$

So if

$\lambda_{\text {max }}\left(A_{i h}\right)<0 \quad \forall i \in\{1, \ldots, n\}$

then the T-S model (6) is globally asymptotically stable. The following theorem describes this sufficient conditions for stability.

\section{Theorem 3}

Suppose that there exist symmetric positive definite matrices $P_{i}$ such that

$A_{i h} P_{i}+P_{i} A_{i h}<0 \quad \forall i \in\{1, \ldots, n\}$

where $A_{i h}$ is the Hermitian matrix related to $A_{i}$, then the global T-S fuzzy model (6) is globally asymptotically stable.

Remark:

Since $\sum_{i=1}^{n} \mu_{i}(z(t))=1$, we note that

$\sum_{i=1}^{n} \mu_{i}(z(t)) \lambda_{\max }\left(A_{i h}\right) \in\left[\min _{i=1}^{n}\left(\lambda_{\max }\left(A_{i h}\right)\right), \max _{i=1}^{n}\left(\lambda_{\max }\left(A_{i h}\right)\right)\right]$

and also, 
$\sum_{i=1}^{n} \mu_{i}(z(t)) \lambda_{\min }\left(A_{i h}\right) \in\left[\min _{i=1}^{n}\left(\lambda_{\min }\left(A_{i h}\right)\right), \max _{i=1}^{n}\left(\lambda_{\min }\left(A_{i h}\right)\right)\right]$

Consequently, (11) implies

$\min _{i=1}^{n}\left(\lambda_{\min }\left(A_{i h}\right)\right) \leq \mathfrak{R} e\left(\lambda_{k}(A(z(t)))\right) \leq \max _{i=1}^{n}\left(\lambda_{\max }\left(A_{i h}\right)\right)$

Obviously, if $\max _{i=1}^{n}\left(\lambda_{\max }\left(A_{i h}\right)\right)<0$ then the system (6) is globally asymptotically stable.

By theorem 2, the imaginary part of the eigenvalues of $A(z(t))$ can be expressed as follows

$$
\left\{\begin{array}{l}
\min \left(\frac{1}{J} \lambda\left(\sum_{i=1}^{n} \mu_{i}(z(t)) A_{i \hbar}\right)\right) \leq \mathfrak{I} m(\lambda(A(z(t)))) \\
\mathfrak{I} m(\lambda(A(z(t)))) \leq \max \left(\frac{1}{J} \lambda\left(\sum_{i=1}^{n} \mu_{i}(z(t)) A_{i \hbar}\right)\right)
\end{array}\right.
$$

Thus the eigenvalues of the $A(z(t))$ matrix belong to the defined rectangle bellow (figure 1), making it possible to bound the dynamic performances of the non-linear system described by the multiple model approach (6).

\section{$\operatorname{Re}(\lambda(A(z)))$}

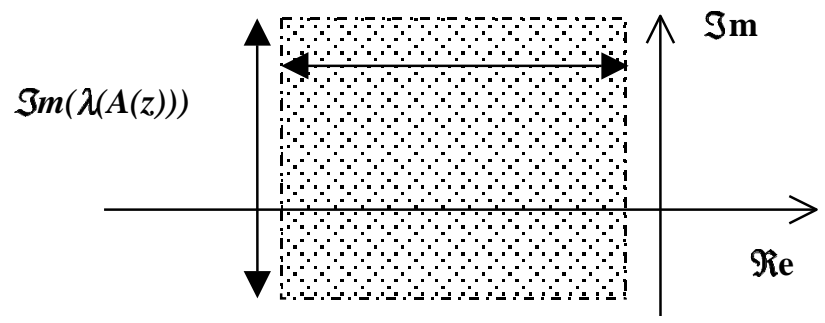

Figure 1. Case where the system (6) is globally asymptotically stable.

\section{Fuzzy controllers design}

\subsection{Stability analysis}

In this section, the PDC technique [1][2][13] is used in order to design fuzzy controllers to stabilise fuzzy system (4). In the PDC approach, the consequent part is local linear feedback law and the antecedent part shares the same fuzzy sets as the fuzzy system.

For the T-S fuzzy controller design, it is supposed that the fuzzy system (4) is locally controllable, i.e. the pairs $\left(A_{i}, B_{i}\right), \forall i \in\{1, \ldots, n\}$ are controllable.

Controller rule i:

$$
\begin{gathered}
\text { IF } z_{1}(t) \text { is } F_{1}^{i} \text { and... and } z_{q}(t) \text { is } F_{q}^{i} \\
\operatorname{THEN~} u(t)=-K_{i} x(t) \quad K_{i} \in \mathbb{R}, \forall i \in\{1, \ldots, n\}
\end{gathered}
$$

The resulting global fuzzy controller, which is nonlinear in general, is:

$u(t)=-\sum_{i=1}^{n} \mu_{i}(z(t)) K_{i} x(t)$

where $\mu_{i}(z(t))$ has to respect constraint (3).

Substituting (12) in (4), we obtain the closed-loop T-S continuous fuzzy system:

$\dot{x}(t)=\tilde{A}(z(t)) x(t)$

where

$$
\begin{aligned}
& \tilde{A}(z(t))=\sum_{i=1}^{n} \sum_{j=1}^{n} \mu_{i}(z(t)) \mu_{j}(z(t)) \tilde{A}_{i j} \\
& \tilde{A}_{i j}=A_{i}-B_{i} K_{j}
\end{aligned}
$$

\section{Theorem 4}

Suppose that there exist symmetric positive definite matrices $P_{i j}$ such that

$\tilde{A}_{i j h} P_{i j}+P_{i j} \tilde{A}_{i j h}<0$

$\forall i, j \in\{1, \ldots, n\} / \mu_{i}(z(t)) \mu_{j}(z(t)) \neq 0$

where $\tilde{A}_{i j h}$ is the Hermitian part of $\tilde{A}_{i j}$. Then the closed loop T-S fuzzy system (13) is globally asymptotically stable.

Proof:

It follows directly from theorem 3 .

Several researches are based on finding a common positive definite matrix [1][2][13][16][18][21], which satisfies a set of LMIs. However there exist a lot of cases, where a common positive definite matrix does not exist, whereas the system is stable.

The proposed method does not require a common positive definite matrix to prove the global asymptotic stability. Thus, if we suppose that there exist $K_{i}$ gains such that the Hermitian matrices $\tilde{A}_{i j h}$ are asymptotically stable, then the closed loop system (13) is globally asymptotically stable.

\subsection{Linearisation for solving BMIs}

Conditions (16) given in theorem 4 and definition (15) lead to solve the following BMIs problem.

$\left(2 A_{i h}-B_{i} K_{j}-K_{j}{ }^{T} B_{i}{ }^{T}\right) P_{i j}+P_{i j}\left(2 A_{i h}-B_{i} K_{j}-K_{j}{ }^{T} B_{i}{ }^{T}\right)<0$

which is BMIs in the variables $P_{i j}$ and $K_{j}$.

We know that BMI problem is not convex and may have multiple local solutions. However, many control problems 
that require the solution to BMIs can be formulated as LMIs, which may be solved very efficiently. Unfortunately, the LMI formulation is very difficult in our case.

In this paper we use the path-following method, developed in [3], for solving BMI problem. This method utilises a first order perturbation approximation to linearize the BMI problem. Hence, the BMIs are converted into a series of LMIs iteratively solved until a desired performance is achieved. Examples are given in [3] [4].

Let $P_{0}$ and $K_{0}$ be initial values such that

$$
P_{i j}=P_{0}+\delta P_{i j}, K_{j}=K_{0}+\delta K_{j}
$$

The BMIs (17) can be rewritten as

$$
\begin{aligned}
& \left(A_{i}+A_{i}^{T}\right)\left(P_{0}+\delta P_{i j}\right)+\left(P_{0}+\delta P_{i j}\right)\left(A_{i}+A_{i}^{T}\right)- \\
& \left(B_{i}\left(K_{0}+\delta K_{j}\right)+\left(K_{0}+\delta K_{j}\right)^{T} B_{i}^{T}\right) P_{0}- \\
& P_{0}\left(B_{i}\left(K_{0}+\delta K_{j}\right)+\left(K_{0}+\delta K_{j}\right)^{T} B_{i}^{T}\right)- \\
& \left(B_{i}\left(K_{0}+\delta K_{j}\right)+\left(K_{0}+\delta K_{j}\right)^{T} B_{i}^{T}\right) \delta P_{i j}- \\
& \delta P_{i j}\left(B_{i}\left(K_{0}+\delta K_{j}\right)+\left(K_{0}+\delta K_{j}\right)^{T} B_{i}^{T}\right)<0
\end{aligned}
$$

Thus, by neglecting the second order terms $B_{i} \delta K_{j} \delta P_{i j}, \delta P_{i j} B_{i} \delta K_{j}$, we get the following LMIs in the variables $\delta P_{i j}$ and $\delta K_{j}$.

$$
\begin{aligned}
& \left\{\begin{array}{l}
P_{0}+\delta P_{i j}>0 \\
2 A_{i h}\left(P_{0}+\delta P_{i j}\right)+2\left(P_{0}+\delta P_{i j}\right) A_{i h}- \\
\left(B_{i}\left(K_{0}+\delta K_{j}\right)+\left(K_{0}+\delta K_{j}\right)^{T} B_{i}^{T}\right) P_{0}- \\
P_{0}\left(B_{i}\left(K_{0}+\delta K_{j}\right)+\left(K_{0}+\delta K_{j}\right)^{T} B_{i}{ }^{T}\right)- \\
\left(B_{i} K_{0}+K_{0}{ }^{T} B_{i}{ }^{T}\right) \delta P_{i j}-\delta P_{i j}\left(B_{i} K_{0}+K_{0}^{T} B_{i}^{T}\right)<0
\end{array}\right. \\
& \forall i, j \in\{1, \ldots, n\} / \mu_{i}(x(t)) \mu_{j}(x(t)) \neq 0
\end{aligned}
$$

It is important to note that the following constraints: $\left\|\delta P_{i j}\right\|<\zeta\left\|P_{0}\right\|$ and $\left\|\delta K_{j}\right\|<\zeta\left\|K_{0}\right\|, 0<\zeta<<1$, must be added in order to ensure that the linear approximation should be valid. For less conservatism it is also possible to take different initial values $\left(P_{i j 0}\right)$ and $\left(K_{j 0}\right)$ instead of common initial values $\left(P_{0}\right)$ and $\left(K_{0}\right)$.

The major weakness of this method is, firstly, the choice of initial value for an acceptable solution and secondly the convergence to a solution which is not guaranteed.

\section{Fuzzy observers design}

The fuzzy controller proposed in section 5 is based on a state feedback. However, in practice, all states of a system are not fully measurable. Thus, the problem addressed in this section is the construction of a fuzzy observer [11][12][2] to estimate states of the T-S fuzzy model (4).
It is supposed that the premise variables are measurable and the T-S fuzzy system ( 4 ) is locally observable, i. e. the pairs $\left(A_{i}, C_{i}\right), \forall i \in\{1, \ldots, n\}$ are observable.

In this case, state observers are designed as follows:

Observer rule i :

IF $z_{1}(t)$ is $F_{l}^{i}$ and... and $z_{q}(t)$ is $F_{q}^{i}$
$\operatorname{THEN}\left\{\begin{array}{l}\dot{\hat{x}}(t)=A_{i} \hat{x}(t)+B_{i} u(t)+L_{i}(y(t)-\hat{y}(t)) \\ \hat{y}(t)=C_{i} \hat{x}(t) \quad \forall i \in\{1, \ldots, n\}\end{array}\right.$

where $\hat{x}(t)$ and $\hat{y}(t)$ denote the estimated state vector and output vector respectively.

The global fuzzy observer for the global T-S fuzzy model (4) is written as follows

$$
\left\{\begin{array}{l}
\dot{\hat{x}}(t)=\sum_{i=1}^{n} \mu_{i}(z(t))\left(A_{i} \hat{x}(t)+B_{i} u(t)+L_{i}(y(t)-\hat{y}(t))\right) \\
\hat{y}(t)=\sum_{i=1}^{n} \mu_{i}(z(t)) C_{i} \hat{x}(t)
\end{array}\right.
$$

The same membership function $\mu_{i}(z(t))$ is used as in the $\mathrm{i}^{\text {th }}$ rule of T-S fuzzy model (4).

Denoting the state estimation error by

$\tilde{x}(t)=x(t)-\hat{x}(t)$

It follows from (4), (5) and (21) that the observer error dynamics is given by the differential equation

$\dot{\tilde{x}}(t)=\sum_{i=1}^{n} \sum_{j=1}^{n} \mu_{i}(z(t)) \mu_{j}(z(t)) \bar{A}_{i j} \tilde{x}(t)$

where $\bar{A}_{i j}=A_{i}-L_{i} C_{j}$

The design of the fuzzy observer consists of determining the local gains $L_{i}$ to ensure the convergence to zero of the error estimation, i.e. the fuzzy observer must satisfy the following condition:

$$
\lim _{t \rightarrow \infty} \tilde{x}(t)=0
$$

\section{Theorem 5}

Suppose that there exists symmetric positive definite matrix $P_{i j}$ such that

$\bar{A}_{i j h} P_{i j}+P_{i j} \bar{A}_{i j h}<0$

$\forall i, j \in\{1, \ldots, n\} / \mu_{i}(x(t)) \mu_{j}(x(t)) \neq 0$

where $\bar{A}_{i j h}$ is the Hermitian part of $\bar{A}_{i j}$. Then there exists a fuzzy observer such that the error estimation (22) is globally asymptotically stable. 
Proof: It follows directly from theorem 3.

For solving the BMIs (24) the same method as in part 5.2 is used, with $L_{i}=L_{0}+\delta L_{i}$ and $P_{i j}$ is as in (18). Thus, we get the following LMIs in the variables $\delta P_{i j}$ and $\delta L_{i}$.

$$
\begin{aligned}
& \left\{\begin{array}{l}
P_{0}+\delta P_{i j}>0 \\
2 A_{i h}\left(P_{0}+\delta P_{i j}\right)+2\left(P_{0}+\delta P_{i j}\right) A_{i h}- \\
\left(\left(L_{0}+\delta L_{i}\right) C_{j}+C_{j}^{T}\left(L_{0}+\delta L_{i}\right)^{T}\right) P_{0}- \\
P_{0}\left(\left(L_{0}+\delta L_{i}\right) C_{j}+C_{j}^{T}\left(L_{0}+\delta L_{i}\right)^{T}\right)- \\
\left(L_{0} C_{j}+C_{j}^{T} L_{0}^{T}\right) \delta P_{i j}-\delta P_{i j}\left(L_{0} C_{j}+C_{j}^{T} L_{0}^{T}\right)<0
\end{array}\right. \\
& \forall i, j \in\{1, \ldots, n\} / \mu_{i}(x(t)) \mu_{j}(x(t)) \neq 0
\end{aligned}
$$

\section{Conclusion}

In this paper we present a new sufficient conditions for the global asymptotic stability of the T-S fuzzy systems. This approach allows stabilising each local model independently and ensures in the same way the global asymptotic stability of the closed loop T-S fuzzy systems. This result classically requires a common quadratic lyapunov function, needs only local quadratic lyapunov functions in the proposed approach. The proposed method will be later extended to the discrete TS fuzzy systems.

\section{References}

[1] Wang H. O., Tanaka K., Griffin M., “ An approach to fuzzy control of nonlinear systems: stability and design issues “ IEEE trans. on Fuzzy Sets, vol. 4, no. 1, pp. 1423, 1996.

[2] Tanaka K., Ikeda T., Wang H. O., "Fuzzy regulators and fuzzy observers: relaxed stability conditions and LMIbased designs “, IEEE trans. on Fuzzy Sets, vol. 6, no. 2, pp. 1-16, 1998.

[3] Hassibi A., How J., Boyd S., “ A path-following method for solving BMI problems in control ", Proc. of ACC, San Diego, California, pp. 1385-1389, Jun 1999.

[4] Hassibi A., How J., Boyd S., " Low-authority controller design via convex optimisation ", Proc. of IEEE CDC, Florida, pp. 140-145, Dec 1998.

[5] Takagi M., Sugeno M., "Fuzzy identification of systems and its application to modelling and control ", IEEE trans. on Systems Man and cybernetics, vol. 15, , no. 1, pp. 116-132, 1988.

[6] Sugeno M., Kang G. T., " Structure identification of fuzzy model “, Fuzzy sets and systems, vol. 28, , pp1533, 1988.

[7] Weinmann, "Uncertain models and robust control", Springer-Verlag, , p. 722, Wien 1991.

[8] Teixeira M. C. M., Zak S. H., "Stabilizing controller design for uncertain nonlinear systems using fuzzy models" IEEE. Trans. on Fuzzy Systems, vol.7, no.2, pp. 133-140, 1999.

[9] Buckley J. J., "Universal fuzzy controllers “, Automatica, vol. 28, no. 6, pp. 1245-1248, 1992.

[10] Narendra K. S., Balakrishnan J., “ A common Lyapunov function for stable LTI systems with commuting Amatrices", IEEE Trans. on Automatic Control, vol. 39, no. 12, pp. 2469-2471, 1994.

[11] Fayaz A. M., " On the Sugeno-type Fuzzy observers “, ACC, Phoenix, Arizona, , pp. 4828-4832, Dec 1999.

[12] Lee K. R. et al., "Observer-based Fuzzy $H^{\infty}$ control for uncertain Nonlinear Systems with Time Delays", ACC, San Diego, California, pp. 1269-1273, Jun 1999.

[13] Tanaka K., Ikeda T., Wang H. O., "Robust stabilisation of uncertain non-linear systems via fuzzy control: quadratic stability, H control theory, and LMIs", IEEE Trans. on Fuzzy Systems, vol. 4, no. 1. pp. 1-12, 1996.

[14] Buckley J. J., "Sugeno type controllers are universal controllers “, Fuzzy sets and systems, no. 53, pp. 299303, 1993.

[15] Chadli M., Maquin D., Ragot J., " Relaxed stability conditions for the T-S fuzzy systems “, Systems Man and cybernetics 2000, Nashville, USA, pp. 3514-3549, Oct. 2000.

[16] Lopez-Toribio C. J., Patton R. J., "T-S fuzzy faulttolerant for a non-linear system ", CDC, Phoenix, Arizona, , pp. 4368-4373, Dec. 1999

[17] Castro J., "Fuzzy logic controllers are universal approximator", IEEE Trans. on Systems, Man, Cybernetics, vol. 25, pp. 629-635, April 1995.

[18] Lopez-Toribio C. J., Patton R. J., Daley S. “ supervisory T-S fuzzy fault-tolerant control of rail traction system “, IFAC, $14^{\text {th }}$ triennial World congress, Beijing, P.R. China. pp. 19-24, 1999.

[19] Tanaka K., Sugeno M., " Stability and design of fuzzy control systems ", Fuzzy Set and Systems, vol. 45, no. 2, pp. 135-156. 1992.

[20] Boyd S. et al., "Linear matrix inequalities in systems and control theory", Philadelphia, PA: SIAM, 1994.

[21] Tanaka K., Nishimuna M., Wang H.O., “ Multi-objective fuzzy control of high rise/high speed elevators using LMIs", ACC, Philadelphia, Pennsylvanie, pp. 34503454, June 1998.

[22] Johansson M., Rantzer A., Arzén K. "Piecewise quadratic stability for affine Sugeno systems", FUZZ. IEEE'98, Anchorage, Alaska, 1998.

[23] Chai J.-S., Tan S., Chan Q., Hang C.-C., “ A general fuzzy control scheme for nonlinear processes with stability analysis", Fuzzy Sets and Systems 100, pp. 179-195, 1998.

[24] Jadbabaie A. "A reduction in conservatism in stability and L2 Gain analysis of T-S fuzzy systems via Linear matrix inequalities", IFAC, $14^{\text {th }}$ triennial World congress, Beijing, P.R. China. pp. 285-289, 1999.

[25] Bromwich T. J. I'A. , "On the roots of the characteristic equation of a linear substution" Acta Mathematica, vol. 30, pp. 297-304, 1906. 